\title{
Parameters Optimization for a Kind of Dynamic Vibration Absorber with Negative Stiffness
}

\author{
Yongjun Shen, Xiaoran Wang, Shaopu Yang, and Haijun Xing \\ Department of Mechanical Engineering, Shijiazhuang Tiedao University, Shijiazhuang 050043, China \\ Correspondence should be addressed to Yongjun Shen; shenyongjun@126.com
}

Received 1 April 2016; Revised 5 July 2016; Accepted 10 July 2016

Academic Editor: Tomasz Kapitaniak

Copyright ( 2016 Yongjun Shen et al. This is an open access article distributed under the Creative Commons Attribution License, which permits unrestricted use, distribution, and reproduction in any medium, provided the original work is properly cited.

\begin{abstract}
A new type of dynamic vibration absorber (DVA) with negative stiffness is studied in detail. At first, the analytical solution of the system is obtained based on the established differential motion equation. Three fixed points are found in the amplitude-frequency curves of the primary system. The design formulae for the optimum tuning ratio and optimum stiffness ratio of DVA are obtained by adjusting the three fixed points to the same height according to the fixed-point theory. Then, the optimum damping ratio is formulated by minimizing the maximum value of the amplitude-frequency curves according to $H_{\infty}$ optimization principle. According to the characteristics of negative stiffness element, the optimum negative stiffness ratio is also established and it could still keep the system stable. In the end, the comparison between the analytical and the numerical solutions verifies the correctness of the analytical solution. The comparisons with three other traditional DVAs under the harmonic and random excitations show that the presented DVA performs better in vibration absorption. This result could provide theoretical basis for optimum parameters design of similar DVAs.
\end{abstract}

\section{Introduction}

Vibration control is becoming important in many engineering practices. One of the common devices for vibration control is dynamic vibration absorber (DVA), which is widely used due to its properties such as efficiency, reliability, and low cost. Researches on DVA had been developed for more than 100 years since the first DVA without damping was invented by Frahm [1] in 1911. In 1928, Ormondroyd and Den Hartog [2] found that a DVA with damping element could suppress the amplitude of primary system in broader frequency range, which had been recognized as the typical Voigt type DVA. Ormondroyd and Den Hartog [2] also found that the amplitude-frequency curves of the primary system with damped DVA would pass through two fixed points which were independent of the absorber damping, and they proposed an optimization criterion to design Voigt type DVA. Hahnkamm [3] derived the optimum natural frequency ratio according to the optimization criterion and described this method as the fixed-point theory. Later, the optimum damping ratio was obtained by Brock [4] in 1946. Currently, the results based on the fixed-point theory have become the classic conclusions which were recorded in vibration engineering textbooks $[5,6]$. However, the conclusions were approximate optimal solutions of Voigt type DVA relative to the exact solutions. Nishihara et al. $[7,8]$ derived the exact series solution for the stiffness and damping coefficient of Voigt type DVA and found that the results according to the fixed-point theory were very close to the exact series solution and the approximate forms were much simpler. Accordingly, the approximate optimization formulae were still used in engineering practices.

To further improve the control performance of DVA, Ren [9] presented a DVA where the damping element was not connected to the primary system but to the earth (a base structure) in 2001. The results indicated it could present better control performance than Voigt type DVA under the same parameter conditions. In 2005, K. Liu and J. Liu [10] got the same result for this DVA by another method. A large number of viscoelastic materials were used in vibration control engineering, and the viscoelastic materials had both damping and stiffness properties. Asami and Nishihara [11, 12] presented a three-element type DVA, which was used to model the viscoelasticity of the devices. They finished 
its optimization design and found the three-element type DVA had better control performance under the same mass ratio. Shen et al. $[13,14]$ studied the approximate analytical solution for four types of semiactive DVAs, and the effects of time delay on the control performance were also investigated.

Stiffness is the ability of resistance to external force. The positive stiffness means that the deformation is in the same direction as the applied external force. On the contrary, the negative stiffness means that the direction of deformation is opposite to that of the applied external force. Researches show that a single bulk solid object with negative stiffness is not stable. Many researches about the properties and stability conditions of the negative stiffness system had been reported in [15-21]. When the positive and negative springs are put in parallel, the system will show nonlinear characteristic near the equilibrium position. The load-bearing capacity of the system with negative stiffness may be better than the positive stiffness and its natural frequency will be reduced simultaneously. The system with negative stiffness has a better vibration control performance when it is stable. Thus, the introduction of negative stiffness to the vibration control system is necessary and meaningful. Platus [22] produced negative stiffness around equilibrium using the buckling of beams with axial load and got an isolation system by combining it with a linear positive spring. Trimboli et al. [23] proposed the application of negative stiffness in mechanical vibration isolator, where the negative stiffness and the positive stiffness springs were arranged in parallel. Park and Luu [24] studied the active control vibration isolator with negative stiffness, and the basic system characteristics were experimentally verified. Mizuno et al. [25-28] studied an active vibration isolation system combining zero-power magnetic suspensions analytically and experimentally. They found that a zero-power system behaved like negative stiffness system and could generate infinite stiffness if it was connected with a normal spring in series. Then, they proposed a new vibration isolation system using negative stiffness which was realized by active control technique [29]. In 2013, Acar and Yilmaz [30] studied a new adaptively passive DVA with a negative stiffness mechanism analytically and experimentally and found that it could suppress the amplitude of the system by appropriately adjusting the parameters. Yang et al. [31] investigated a nonlinear vibration isolation system with a negative stiffness mechanism. Zhu et al. [32] invented a kind of negative stiffness system used in gravity compensation for tiny vibration machines. In 2015, Li et al. [33] studied a kind of passively negative stiffness device based on preload of three springs. Many scholars had studied the application of negative stiffness in vibration isolation system for its advantages, but little work has been done about the optimal parameters of the DVA with negative stiffness. In 2015, Peng et al. [34] studied a new type of DVA with negative stiffness, finished its parameter optimization, and found that the DVA with negative stiffness had good control performance.

In this paper, the effect of negative stiffness on the amplitude of the primary system is studied by introducing the negative stiffness connected to the grounded

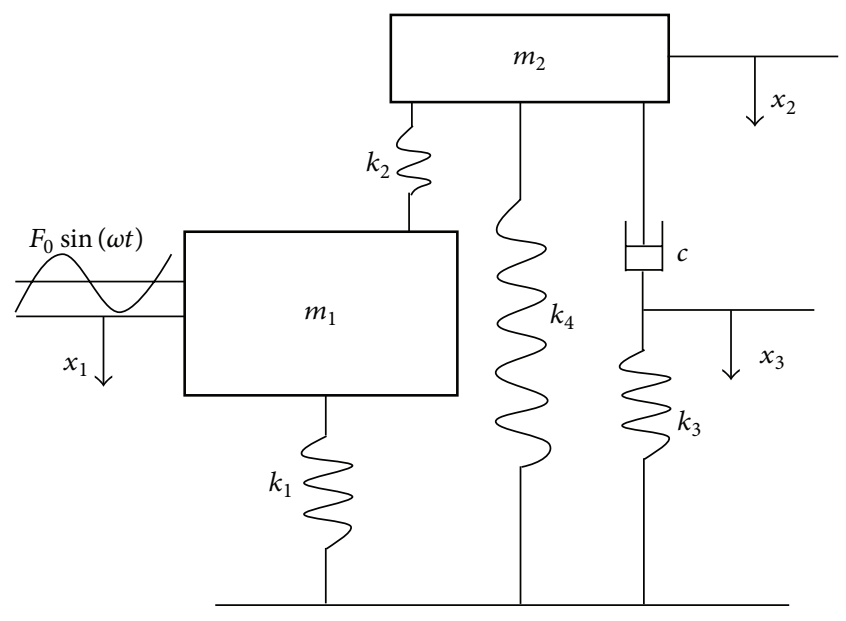

FIGURE 1: The model of dynamic vibration absorber.

three-element type DVA. The model is established and the optimum parameters are obtained based on the fixed-point theory in Section 2. The comparison of the analytical solution with the numerical one is fulfilled, and the result shows that the analytical solution is correct and satisfactorily precise. Then, the presented DVA is compared with other existing DVAs under harmonic excitation in Section 3 and random excitation in Section 4. It could be concluded that the system with the DVA in this paper has better control performance than the traditional Voigt type DVA, the DVA by Ren, and the DVA by Asami, whenever the primary system is subjected to harmonic or random excitations. This research may provide a new theoretical basis for designing new type of DVAs.

\section{The Model of DVA and Parameters Optimization}

2.1. The Analytical Solution. The grounded three-element type DVA with negative stiffness is shown in Figure 1.

According to Newtonian's second law, the differential motion equation can be established as

$$
\begin{aligned}
& m_{1} \ddot{x}_{1}+k_{1} x_{1}+k_{2}\left(x_{1}-x_{2}\right)=F_{0} \sin (\omega t), \\
& m_{2} \ddot{x}_{2}+k_{2}\left(x_{2}-x_{1}\right)+c\left(\dot{x}_{2}-\dot{x}_{3}\right)+k_{4} x_{2}=0, \\
& c\left(\dot{x}_{3}-\dot{x}_{2}\right)+k_{3} x_{3}=0,
\end{aligned}
$$

where $m_{1}, m_{2}, k_{1}$, and $k_{2}$ are the masses and linear stiffness coefficients of the primary system and DVA, respectively. $k_{4}$ is the coefficient of negative stiffness spring which is connected to the earth directly; $k_{3}$ and $c$ are linear stiffness and damping coefficient of viscoelastic Maxwell model which are origination of three-element model. $x_{1}, x_{2}$, and $x_{3}$ are the displacement of the primary system, the absorber, and the split point of spring and damping in Maxwell model, respectively. $F_{0}$ and $\omega$ are the amplitude and frequency of the force excitation. 
Using the following parametric transformation

$$
\begin{aligned}
\omega_{1} & =\sqrt{\frac{k_{1}}{m_{1}}}, \\
\omega_{2} & =\sqrt{\frac{k_{2}}{m_{2}}}, \\
\xi & =\frac{c}{2 m_{2} \omega_{2}}, \\
\mu & =\frac{m_{2}}{m_{1}}, \\
\alpha_{1} & =\frac{k_{3}}{k_{2}}, \\
\alpha_{2} & =\frac{k_{4}}{k_{2}}, \\
f & =\frac{F_{0}}{m_{1}},
\end{aligned}
$$

(1) becomes

$$
\begin{aligned}
& \ddot{x}_{1}+\omega_{1}^{2} x_{1}+\mu \omega_{2}^{2}\left(x_{1}-x_{2}\right)=f \sin (\omega t), \\
& \ddot{x}_{2}+\omega_{2}^{2}\left(x_{2}-x_{1}\right)+2 \xi \omega_{2}\left(\dot{x}_{2}-\dot{x}_{3}\right)+\alpha_{2} \omega_{2}^{2} x_{2}=0, \\
& 2 \xi \omega_{2}\left(\dot{x}_{3}-\dot{x}_{2}\right)+\alpha_{1} \omega_{2}^{2} x_{3}=0 .
\end{aligned}
$$

Letting $f \sin (\omega t)$ in (3) be represented by $f e^{j \omega t}$, the steadystate solutions take the forms as

$$
\begin{aligned}
& x_{1}=X_{1} e^{j \omega t}, \\
& x_{2}=X_{2} e^{j \omega t}, \\
& x_{3}=X_{3} e^{j \omega t} .
\end{aligned}
$$

Substituting (4) into (3), one can obtain

$$
X_{1}=\frac{f\left(j A_{1}+B_{1}\right)}{j C_{1}+D_{1}},
$$

where $j$ is the imaginary unit and the other parameters are as follows:

$$
\begin{aligned}
A_{1} & =-2 \xi \omega\left[\omega^{2}-\left(1+\alpha_{1}+\alpha_{2}\right) \omega_{2}^{2}\right] \\
B_{1} & =\alpha_{1}\left(1+\alpha_{2}\right) \omega_{2}^{3}-\alpha_{1} \omega^{2} \omega_{2}, \\
C_{1} & =2 \xi \omega\left\{\omega^{4}+\left(1+\alpha_{1}+\alpha_{2}\right) \omega_{1}^{2} \omega_{2}^{2}+\mu\left(\alpha_{1}+\alpha_{2}\right) \omega_{2}^{4}\right. \\
& \left.+\omega^{2}\left[-\omega_{1}^{2}-\left(1+\alpha_{1}+\alpha_{2}+\mu\right) \omega_{2}^{2}\right]\right\}
\end{aligned}
$$

$$
\begin{aligned}
D_{1} & =\alpha_{1} \omega_{2}\left\{\omega^{4}+\left(1+\alpha_{2}\right) \omega_{1}^{2} \omega_{2}^{2}+\mu \alpha_{2} \omega_{2}^{4}\right. \\
& \left.-\omega^{2}\left[\omega_{1}^{2}+\left(1+\alpha_{2}+\mu\right) \omega_{2}^{2}\right]\right\} .
\end{aligned}
$$

Introducing the parameters

$$
\begin{aligned}
\lambda & =\frac{\omega}{\omega_{1}} \\
v & =\frac{\omega_{2}}{\omega_{1}} \\
\delta_{\text {st }} & =\frac{F_{0}}{k_{1}}
\end{aligned}
$$

and supposing $A$ is the normalized amplitude amplification factor of the primary system, one could obtain

$$
A^{2}=\left|\frac{X_{1}}{\delta_{\text {st }}}\right|^{2}=\frac{\xi^{2} A_{2}^{2}+B_{2}^{2}}{\xi^{2} C_{2}^{2}+D_{2}^{2}},
$$

where

$$
\begin{aligned}
A_{2} & =2 \lambda\left[\left(1+\alpha_{1}+\alpha_{2}\right) v^{2}-\lambda^{2}\right], \\
B_{2} & =\alpha_{1}\left(1+\alpha_{2}\right) v^{3}-\alpha_{1} \lambda^{2} v, \\
C_{2} & =2 \lambda\left\{\lambda^{4}+\left(1+\alpha_{1}+\alpha_{2}\right) v^{2}+\mu\left(\alpha_{1}+\alpha_{2}\right) v^{4}\right. \\
& \left.+\lambda^{2}\left[-1-\left(1+\alpha_{1}+\alpha_{2}+\mu\right) v^{2}\right]\right\}, \\
D_{2} & =\alpha_{1} v\left\{\lambda^{4}+\left(1+\alpha_{2}\right) v^{2}+\mu \alpha_{2} v^{4}\right. \\
& \left.-\lambda^{2}\left[1+\left(1+\alpha_{2}+\mu\right) v^{2}\right]\right\} .
\end{aligned}
$$

2.2. Parameters Optimization. By simple deduction of (8), it can be found that the normalized amplitude-frequency curve will pass through three points which are independent of the damping ratio. Some normalized amplitude-frequency curves under different damping ratios as $0.4,0.5$, and 0.9 are shown in Figure 2. It could be clearly seen that there exist three common fixed points $P, Q$, and $R$ on all the curves, which are independent of the damping ratio. In order to find the values of the fixed points, the following equation should hold:

$$
\frac{A_{2}}{C_{2}}=\frac{B_{2}}{D_{2}} .
$$

Substituting (9) into (10), one can get

$$
\lambda^{6}+q_{1} \lambda^{4}+q_{2} \lambda^{2}+q_{3}=0
$$


where

$$
\begin{aligned}
& q_{1}=-\left[1+\left(2+\alpha_{1}+2 \alpha_{2}+\mu\right) v^{2}\right] \\
& q_{2}=\left(2+\alpha_{1}+2 \alpha_{2}\right) v^{2}+\left[1+\mu+\alpha_{1}\left(1+\alpha_{2}+\mu\right)+\alpha_{2}\left(2+\alpha_{2}+2 \mu\right) v^{4}\right] \\
& q_{3}=\frac{-\left\{2\left(1+\alpha_{2}\right)\left(1+\alpha_{1}+\alpha_{2}\right) v^{4}+\left[\alpha_{1}+2 \alpha_{1} \alpha_{2}+2 \alpha_{2}\left(1+\alpha_{2}\right)\right] \mu v^{6}\right\}}{2}
\end{aligned}
$$

Because the three fixed points have nothing to do with the when $\xi=0$, and damping ratio, one could obtain

$$
\begin{aligned}
& |A|=\left|\frac{X_{1}}{\delta_{\text {st }}}\right| \\
& =\left|\frac{\left(1+\alpha_{2}\right) v^{2}-\lambda^{2}}{\lambda^{4}+\left(1+\alpha_{2}\right) v^{2}+\mu \alpha_{2} v^{4}-\lambda^{2}\left[1+\left(1+\alpha_{2}+\mu\right) v^{2}\right]}\right|, \begin{array}{l}
\text { (13) } \\
\qquad|A|=\left|\frac{X_{1}}{\delta_{\text {st }}}\right|=\left|\frac{\left(1+\alpha_{1}+\alpha_{2}\right) v^{2}-\lambda^{2}}{\lambda^{4}+\left(1+\alpha_{1}+\alpha_{2}\right) v^{2}+\mu\left(\alpha_{1}+\alpha_{2}\right) v^{4}+\lambda^{2}\left[-1-\left(1+\alpha_{1}+\alpha_{2}+\mu\right) v^{2}\right]}\right|,
\end{array}
\end{aligned}
$$

when $\xi=\infty$.

Accordingly, one can get

$$
|A|=\left|\frac{X_{1}}{\delta_{\text {st }}}\right|=\left|\frac{\left(2+2 \alpha_{2}+\alpha_{1}\right) v^{2}-2 \lambda^{2}}{\alpha_{1} v^{2}+\alpha_{1} \mu v^{4}-\alpha_{1} v^{2} \lambda^{2}}\right| .
$$

For simplicity, the three real roots of (11) are supposed as $\lambda_{P}^{2}$, $\lambda_{Q}^{2}$, and $\lambda_{R}^{2}$. Once the values of $\lambda_{P}, \lambda_{\mathrm{Q}}$, and $\lambda_{R}$ are determined, the ordinates in Figure 2 of the three fixed points could be obtained:

$$
\begin{aligned}
& \left|\frac{X_{1}}{\delta_{\text {st }}}\right|_{P}=\frac{\left(2+2 \alpha_{2}+\alpha_{1}\right) v^{2}-2 \lambda_{P}^{2}}{\alpha_{1} v^{2}+\alpha_{1} \mu v^{4}-\alpha_{1} v^{2} \lambda_{P}^{2}} \\
& \left|\frac{X_{1}}{\delta_{\text {st }}}\right|_{Q}=-\frac{\left(2+2 \alpha_{2}+\alpha_{1}\right) v^{2}-2 \lambda_{Q}^{2}}{\alpha_{1} v^{2}+\alpha_{1} \mu v^{4}-\alpha_{1} v^{2} \lambda_{Q}^{2}} \\
& \left|\frac{X_{1}}{\delta_{\text {st }}}\right|_{R}=\frac{\left(2+2 \alpha_{2}+\alpha_{1}\right) v^{2}-2 \lambda_{R}^{2}}{\alpha_{1} v^{2}+\alpha_{1} \mu v^{4}-\alpha_{1} v^{2} \lambda_{R}^{2}}
\end{aligned}
$$

Optimum tuning of DVA will be accomplished with the condition that the three fixed points are adjusted to equal height. At first, by adjusting the fixed points $P$ and $R$ to equal height, that is to say, $\left|X_{1} / \delta_{\text {st }}\right|_{P}=\left|X_{1} / \delta_{\text {st }}\right|_{R}$, one could obtain

$$
\alpha_{1}=2\left(-1-\alpha_{2}+\mu+\frac{1}{v^{2}}\right)
$$

Substituting (17) into (11), one can get

$$
\begin{aligned}
& \left(1+\mu v^{2}-\lambda^{2}\right)\left\{2\left(1+\alpha_{2}\right) v^{2}\right. \\
& +\left[-\left(1+\alpha_{2}\right)^{2}+\mu+2 \alpha_{2} \mu\right] v^{4}-2\left(1+\mu v^{2}\right) \lambda^{2} \\
& \left.+\lambda^{4}\right\}=0 .
\end{aligned}
$$

Solving (18) for $\lambda^{2}$ yields

$$
\begin{aligned}
\lambda_{P}^{2} & =1+\mu v^{2} \\
& -\sqrt{1-2\left(1+\alpha_{2}-\mu\right) v^{2}+\left[\left(1+\alpha_{2}\right)^{2}-\mu\left(1+\alpha_{2}\right)+\mu^{2}\right] v^{4}}, \\
\lambda_{Q}^{2} & =1+\mu v^{2}, \\
\lambda_{R}^{2} & =1+\mu v^{2} \\
& +\sqrt{1-2\left(1+\alpha_{2}-\mu\right) v^{2}+\left[\left(1+\alpha_{2}\right)^{2}-\mu\left(1+\alpha_{2}\right)+\mu^{2}\right] v^{4}} .
\end{aligned}
$$

Then, (16) can be written as

$$
\begin{gathered}
\left|\frac{X_{1}}{\delta_{\mathrm{st}}}\right|_{P, R}=\frac{1}{1-\left(1+\alpha_{2}-\mu\right) v^{2}}, \\
\left|\frac{X_{1}}{\delta_{\mathrm{st}}}\right|_{\mathrm{Q}}=\frac{1+\left(-1-\alpha_{2}+\mu\right) v^{2}}{\mu v^{4}} .
\end{gathered}
$$




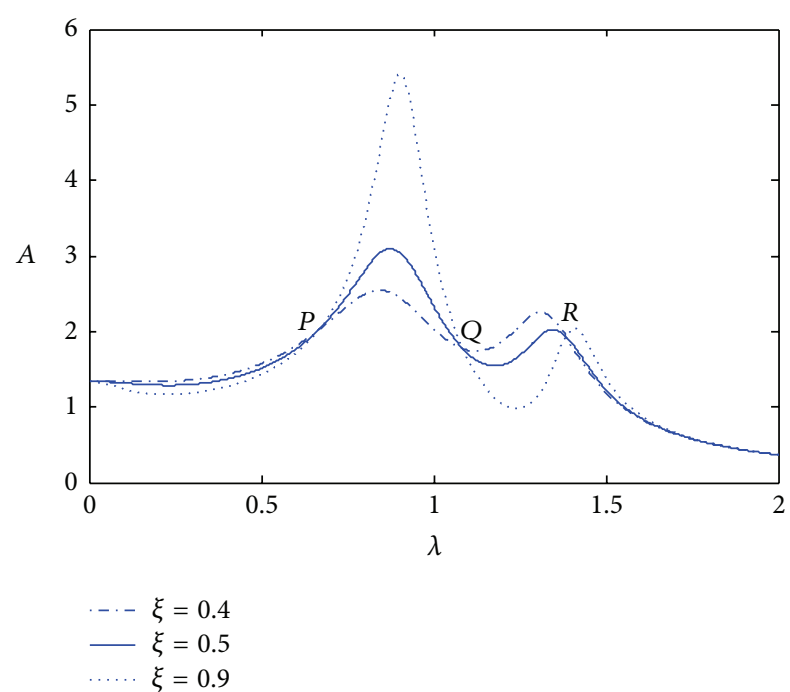

Figure 2: The amplitude-frequency curves with parameters as $\mu=$ $0.1, v=1.3, \alpha_{1}=0.6$, and $\alpha_{2}=-0.6$.

Secondly, adjusting the points $P$ (or $R$ ) and $Q$ to equal height, that is to say, $\left|X_{1} / \delta_{\text {st }}\right|_{P, R}=\left|X_{1} / \delta_{\text {st }}\right|_{Q}$ in (20), one can obtain the optimum tuning condition with respect to $v$ :

$$
v_{\mathrm{opt}}=\sqrt{\frac{1}{1+\alpha_{2}+\sqrt{\mu}-\mu}} .
$$

Substituting (21) into (17), the optimum value of $\alpha_{1}$ could be established:

$$
\alpha_{1 \mathrm{opt}}=2 \sqrt{\mu}
$$

At the fixed points, the normalized amplitude amplification factor of the primary system will be

$$
\left|\frac{X_{1}}{\delta_{\mathrm{st}}}\right|_{P, \mathrm{Q}, \mathrm{R}}=\frac{1+\alpha_{2}+\sqrt{\mu}-\mu}{\sqrt{\mu}} .
$$

For the condition of the three fixed points with equal height, optimum damping ratio $\xi_{\text {opt }}$ is achieved when the two resonance peaks are adjusted to equal height, as shown in Figure 3.

In order to obtain the optimum damping ratio, one should know the abscissa values at the two resonance peaks, namely, $\lambda_{1,2}$. That means

$$
\begin{aligned}
& \frac{\partial A}{\partial \lambda_{1}}=0 \\
& \frac{\partial A}{\partial \lambda_{2}}=0 .
\end{aligned}
$$

Substituting (8) into (24), the values of $\xi_{1}$ and $\xi_{2}$ can be obtained. Then, one could get $\xi_{\text {opt }}=\left(\xi_{1}+\xi_{2}\right) / 2$ through the similar way by Den Hartog [5]. However, it is difficult to obtain the analytical results due to the complicated forms in (24).

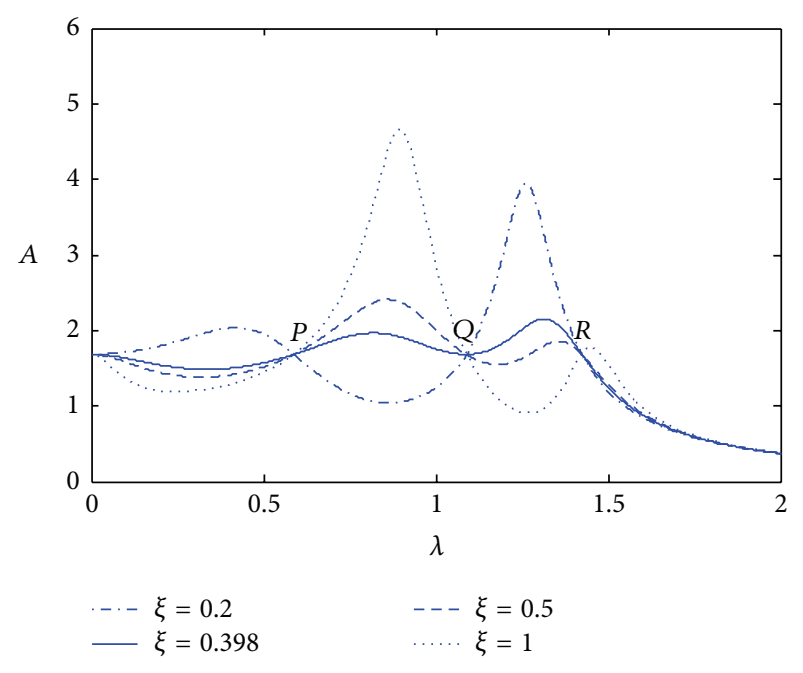

FIgURE 3: The amplitude-frequency curve with parameters as $\mu=$ $0.1, v=1.370, \alpha_{1}=0.632$, and $\alpha_{2}=-0.684$.

When the two resonance peaks are at the same height, one could observe that the point $Q$ is almost the area where the slope of the amplitude-frequency curve is zero. The abscissa value of point $Q$ in (19) has been obtained, so that the approximate optimum damping ratio can be solved according to the abscissa value of point $Q$; namely,

$$
\begin{aligned}
\frac{\partial A^{2}}{\partial \lambda^{2}} & =0, \\
\alpha_{1} & =2 \sqrt{\mu}, \\
v & =\sqrt{\frac{1}{1+\alpha_{2}+\sqrt{\mu}-\mu},} \\
\lambda_{Q}^{2} & =1+\mu v^{2} .
\end{aligned}
$$

Solving (25), one can get

$$
\frac{\sqrt{\mu}\left[-\xi^{4}\left(1+\alpha_{2}+\sqrt{\mu}\right)^{2}+\mu^{2}\right]}{1+\alpha_{2}+\sqrt{\mu}-\mu}=0 .
$$

Furthermore, one could obtain

$$
\xi_{\mathrm{opt}} \cong \sqrt{\frac{\mu}{1+\alpha_{2}+\sqrt{\mu}}} .
$$

At this point, there still exists an adjustable parameter $\alpha_{2}$ in the optimum natural frequency ratio and damping ratio. It is clear that a negative $\alpha_{2}$ will make the three fixed points much lower; that means one can get a better vibration control performance. According to the characteristic of negative stiffness, it can be achieved if the negative stiffness material is applied by preload. The preload will cause predisplacement of the primary system, so that an approximation is adopted which makes the predisplacement equal to the values of the 
three fixed points. That means the response value to zerofrequency excitation is equal to that of the three fixed points; that is,

$$
\left|\frac{X_{1}}{\delta_{\mathrm{st}}}\right|_{\lambda=0}=\left|\frac{X_{1}}{\delta_{\mathrm{st}}}\right|_{P, \mathrm{Q}, R}
$$

Accordingly,

$$
\begin{aligned}
& \sqrt{\frac{\left(1+\alpha_{2}\right)^{2}}{\left(1+\alpha_{2}+\mu \alpha_{2} /\left(1+\alpha_{2}+\sqrt{\mu}-\mu\right)\right)^{2}}} \\
& \quad=\sqrt{\frac{\left(1+\alpha_{2}+\sqrt{\mu}-\mu\right)^{2}}{\mu}} .
\end{aligned}
$$

Solving (29), one can get the negative stiffness ratio as

$$
\begin{aligned}
& \alpha_{2 a}=-1-\sqrt{\mu}, \\
& \alpha_{2 b}=-1+\sqrt{\mu}, \\
& \alpha_{2 c}=-1-(1+\sqrt{2}) \sqrt{\mu}, \\
& \alpha_{2 d}=-1+(-1+\sqrt{2}) \sqrt{\mu}, \\
& \alpha_{2 e}=-1-\sqrt{\mu}+\mu .
\end{aligned}
$$

When the possible values in (30) are taken into the other optimization parameters and the normalized amplitude amplification factor of the primary system, one could find that only $\alpha_{2 b}$ can ensure the system stability and the other four possible values will cause the system to be unstable or make optimum damping ratio negative. Obviously, these four values are meaningless, so that only $\alpha_{2 b}$ is taken as the optimum negative stiffness ratio:

$$
\alpha_{2 \mathrm{opt}}=\alpha_{2 b}=-1+\sqrt{\mu} .
$$

At this time, all the optimal parameters of DVA in this paper are obtained as

$$
\begin{aligned}
v_{\mathrm{opt}} & =\sqrt{\frac{1}{2 \sqrt{\mu}-\mu}}, \\
\alpha_{1 \mathrm{opt}} & =2 \sqrt{\mu}, \\
\xi_{\mathrm{opt}} & \cong\left(\frac{\mu}{4}\right)^{1 / 4} .
\end{aligned}
$$

As a comparison and summarization, the models by Den Hartog et al. $[5,9,11]$ are presented in Figure 4. All the optimal parameters of the four models are summarized in Table 1.

Figure 5 shows the amplitude-frequency curve under the optimum parameters above, which is denoted by the solid line. It can be found that the optimization purposes are basically achieved. In order to illustrate the correctness and precision of the analytical solution, the numerical solution is also presented. In the numerical investigation, the fourorder Runge-Kutta method is used to solve (3). Here, the

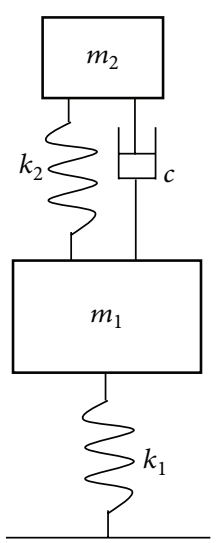

(a) The model by Den Hartog

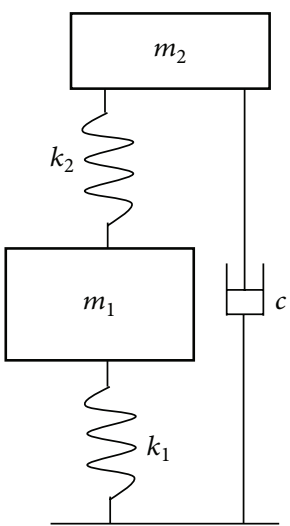

(b) The model by Ren

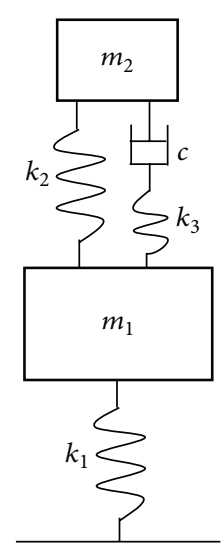

(c) The model by Asami
Figure 4: The models of DVAs.

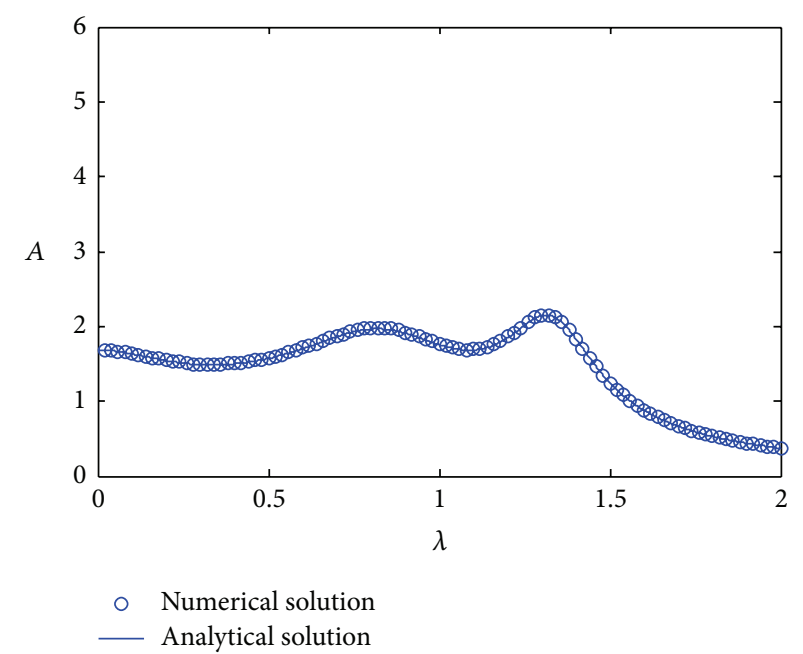

FIGURE 5: The amplitude-frequency curve with the optimum parameters as $\mu=0.1, v=1.370, \alpha_{1}=0.632, \alpha_{2}=-0.684$, and $\xi=0.398$.

total computation time is 100 times of the excitation period, and the maximum value of the later quarter of the time response is considered as the steady-state amplitude. From the observation, it can be concluded that the analytical and the numerical solution agree very well with each other.

\section{Comparison with Other DVAs}

In order to illustrate the control performance of the presented DVA with negative stiffness, the normalized amplitudefrequency curves of other typical DVAs proposed by Den Hartog et al. are also presented. Based on the same mass ratio $\mu=0.1$, the other three curves are obtained based on the optimum parameters they presented $[5,9,11]$. Table 2 shows the values of optimal parameters used in presented comparison when $\mu=0.1$. The four curves are shown in Figure 6 and denoted by dotted line, dash line, dash dot line, and solid line, respectively.

From the comparison, it can be concluded that the DVA in this paper can not only suppress the amplitude of the 
TABLE 1: The formulas of Den Hartog, Ren, and Asami realizations of DVAs.

\begin{tabular}{|c|c|c|c|c|}
\hline Number & The model of DVAs & Optimum tuning & Optimum damping & Optimum negative stiffness \\
\hline 1 & Den Hartog & $v_{\mathrm{opt}}=\frac{1}{1+\mu}$ & $\xi_{\mathrm{opt}}=\sqrt{\frac{3 \mu}{8(1+\mu)}}$ & \\
\hline 2 & Ren & $v_{\mathrm{opt}}=\sqrt{\frac{1}{1-\mu}}$ & $\xi_{\mathrm{opt}}=\sqrt{\frac{3 \mu}{8(1-0.5 \mu)}}$ & \\
\hline 3 & Asami & $\begin{array}{l}v_{\mathrm{opt}}=\sqrt{\frac{1}{1+\mu}\left(1-\sqrt{\frac{\mu}{1+\mu}}\right)} \\
\alpha_{1 \mathrm{opt}}=2[\mu+\sqrt{\mu(1+\mu)}]\end{array}$ & $\begin{array}{l}\xi_{\mathrm{opt}} \cong \sqrt{\frac{1+r}{r} \cdot \frac{-b-\sqrt{b^{2}-a c}}{a}} \\
a=-2-2 r+5 r^{2}+4 r^{3}-2 r^{5}+r^{6} \\
b=2-3 r^{2}-r^{4} \\
c=-2+2 r+r^{2} \\
r=\sqrt{(1+\mu) / \mu}\end{array}$ & \\
\hline 4 & $\begin{array}{l}\text { The model in this } \\
\text { paper }\end{array}$ & $\begin{array}{l}v_{\mathrm{opt}}=\sqrt{\frac{1}{2 \sqrt{\mu}-\mu}} \\
\alpha_{1 \mathrm{opt}}=2 \sqrt{\mu}\end{array}$ & $\xi_{\mathrm{opt}} \cong\left(\frac{\mu}{4}\right)^{1 / 4}$ & $\alpha_{2 \mathrm{opt}}=-1+\sqrt{\mu}$ \\
\hline
\end{tabular}

TABLE 2: The values of parameters used in presented comparison when $\mu=0.1$.

\begin{tabular}{|c|c|c|c|c|}
\hline Number & The model of DVAs & Optimum tuning & Optimum damping & Optimum negative stiffness \\
\hline 1 & Den Hartog & $v_{\text {opt }}=0.909$ & $\xi_{\text {opt }}=0.185$ & \\
\hline 2 & Ren & $v_{\mathrm{opt}}=1.054$ & $\xi_{\mathrm{opt}}=0.199$ & \\
\hline 3 & Asami & $\begin{array}{c}v_{\mathrm{opt}}=0.797 \\
\alpha_{1 \mathrm{opt}}=0.863\end{array}$ & $\xi_{\mathrm{opt}}=0.282$ & \\
\hline 4 & $\begin{array}{l}\text { The model in this } \\
\text { paper }\end{array}$ & $\begin{array}{c}v_{\mathrm{opt}}=1.370 \\
\alpha_{1 \mathrm{opt}}=0.632\end{array}$ & $\xi_{\mathrm{opt}}=0.398$ & $\alpha_{2 \mathrm{opt}}=-0.684$ \\
\hline
\end{tabular}

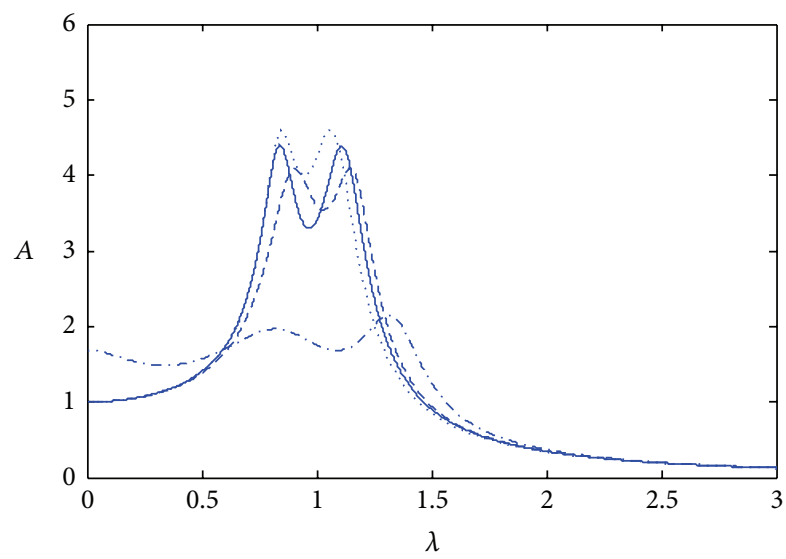

..... The result by Den Hartog _ T The result by Asami -- The result by Ren _..- Our result

FIgURE 6: The comparison with other DVAs under $\mu=0.1$.

primary system in resonance region significantly but also extend the efficient frequency range of vibration absorption.

\section{The Response of the Primary System to Random Excitation}

The primary system is usually subject to random excitation in practical engineering, so that it is very important and meaningful to investigate the system response to random excitation. When the primary system is subjected to random excitation with zero mean and the power spectral density as $S(\omega)=S_{0}$, the power spectral density functions of the absolute displacement response of the negative stiffness model and the other three typical DVAs model are

$$
\begin{aligned}
& S_{N}(\omega)=\left|X_{1 N}\right|^{2} S_{0}, \\
& S_{V}(\omega)=\left|X_{1 V}\right|^{2} S_{0}, \\
& S_{R}(\omega)=\left|X_{1 R}\right|^{2} S_{0}, \\
& S_{A}(\omega)=\left|X_{1 A}\right|^{2} S_{0},
\end{aligned}
$$

where the subscripts $N, V, R$, and $A$ represent the negative stiffness model, the Voigt type DVA by Den Hartog, and the model by Ren and Asami, respectively. Peng et al. [34] had presented the mean square response of the primary system of the Voigt type DVA and the DVA by Ren; namely,

$$
\begin{aligned}
& \sigma_{V}^{2}=\int_{-\infty}^{\infty} S_{V}(\omega) d \omega=S_{0} \int_{-\infty}^{\infty}\left|X_{1 V}\right|^{2} d \omega=\frac{\pi S_{0} Y_{1}}{2 \omega_{1}^{3} \mu \xi v}, \\
& \sigma_{R}^{2}=\int_{-\infty}^{\infty} S_{R}(\omega) d \omega=S_{0} \int_{-\infty}^{\infty}\left|X_{1 R}\right|^{2} d \omega=\frac{\pi S_{0} Y_{2}}{2 \omega_{1}^{3} \mu \xi v^{5}},
\end{aligned}
$$


where

$$
\begin{aligned}
& Y_{1}=1+v^{4}(1+\mu)^{2}+v^{2}\left(4 \mu \xi^{2}+4 \xi^{2}-\mu-2\right), \\
& Y_{2}=1+v^{4}+\left(\mu+4 \xi^{2}-2\right) .
\end{aligned}
$$

The mean square response of the primary system of the DVA in this paper and the DVA by Asami can be deduced as

$$
\begin{aligned}
\sigma_{N}^{2} & =\int_{-\infty}^{\infty} S_{N}(\omega) d \omega=S_{0} \int_{-\infty}^{\infty}\left|X_{1 N}\right|^{2} d \omega \\
& =\frac{\pi S_{0} Y_{3}}{2 \omega_{1}^{3} \mu \xi v^{7} \alpha_{1}^{2}\left(1+\alpha_{2}+\mu \alpha_{2} v^{2}\right)}, \\
\sigma_{A}^{2} & =\int_{-\infty}^{\infty} S_{A}(\omega) d \omega=S_{0} \int_{-\infty}^{\infty}\left|X_{1 A}\right|^{2} d \omega \\
& =\frac{\pi S_{0} Y_{4}}{2 \omega_{1}^{3} \mu \xi k^{2} v^{3}},
\end{aligned}
$$

where

$$
\begin{aligned}
Y_{3} & =4 \xi^{2}\left(1+\alpha_{2}+\alpha_{2} \mu v^{2}\right)\left\{1-2\left(1+\alpha_{1}+\alpha_{2}-\mu\right) v^{2}\right. \\
& \left.+\left[\left(1+\alpha_{1}+\alpha_{2}\right)^{2}-\left(1+2 \alpha_{1}+2 \alpha_{2}\right) \mu+\mu^{2}\right] v^{4}\right\} \\
& +\alpha_{1}^{2} v^{2}\left\{1+\alpha_{2}+\left[-2\left(1+\alpha_{2}\right)^{2}+\mu+2 \mu \alpha_{2}\right] v^{2}\right. \\
& \left.+\left[\left(1+\alpha_{2}\right)^{3}-2 \alpha_{2}\left(1+\alpha_{2}\right) \mu+\alpha_{2} \mu^{2}\right] v^{4}\right\}, \\
Y_{4} & =k^{2} v^{2}\left[1-(2+\mu) v^{2}+(1+\mu)^{2} v^{4}\right]+4 \xi^{2}\{1 \\
& \left.+(1+k) v^{2}\left[-2+(1+k)(1+\mu) v^{2}\right]\right\} .
\end{aligned}
$$

According to Table 2, the mean square response of the primary system under the optimal parameters can be obtained, respectively, when $\mu=0.1$ :

$$
\begin{aligned}
\sigma_{N}^{2} & =\frac{3.091 \pi S_{0}}{\omega_{1}^{3}}, \\
\sigma_{V}^{2} & =\frac{6.401 \pi S_{0}}{\omega_{1}^{3}}, \\
\sigma_{R}^{2} & =\frac{5.780 \pi S_{0}}{\omega_{1}^{3}}, \\
\sigma_{A}^{2} & =\frac{6.030 \pi S_{0}}{\omega_{1}^{3}} .
\end{aligned}
$$

The results show that the DVA with negative stiffness can still present better performance than the other DVAs even under the random excitation.

In order to get more realistic results, 50-second random excitation is constructed, which is composed of 5000 normalized random numbers with zero mean value and unit variance, respectively. The time history of the random excitation is shown in Figure 7. Here, we take the primary mass $m_{1}=1 \mathrm{~kg}$ and stiffness of the primary system $k_{1}=100 \mathrm{~N} / \mathrm{m}$. Then, the other parameters can be calculated according to

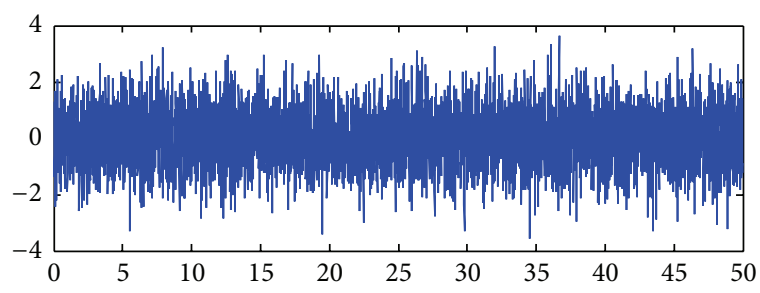

FIGURE 7: The time history of the random excitation.

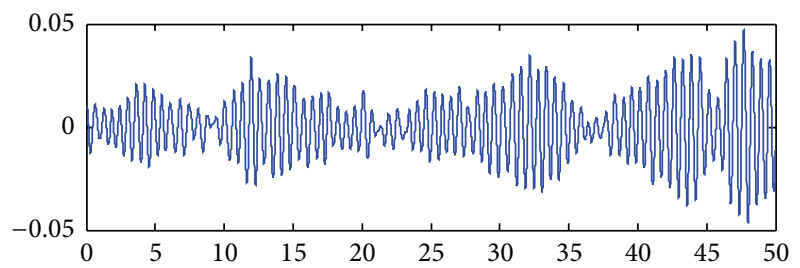

FIGURE 8: The time history of the primary system without DVA.

TABLE 3: The variances and decrease ratios of the displacement of the primary system.

\begin{tabular}{lcc}
\hline Model of DVA & Variances & Decrease ratios (\%) \\
\hline Without DVA & $2.0083 e-04$ & \\
DVA by Den Hartog & $3.1235 e-05$ & 84.45 \\
DVA by Ren & $2.6979 e-05$ & 86.57 \\
DVA by Asami & $2.9025 e-05$ & 85.55 \\
Our result & $1.2334 e-05$ & 93.86 \\
\hline
\end{tabular}

Table 1. And the parameters for the DVA proposed in this paper can be calculated according to (31) and (32). Based on the fourth-order Runge-Kutta method, the response of the primary system without DVA and with different DVAs can be obtained. The time history of these primary systems is shown in Figures 8-12. The response variances and decrease ratios of the primary system for the different systems are summarized in Table 3.

\section{Conclusions}

A new type of dynamic vibration absorber with negative stiffness is investigated. The study shows that there still exist three fixed points on the amplitude-frequency curves. Based on the fixed-point theory, the optimum tuning ratio, the optimum stiffness ratio, and the optimum damping ratio are obtained. The method to determine the optimum negative stiffness ratio is proposed, and accordingly the optimum negative stiffness ratio is determined. Then, the four-order Runge-Kutta method is used to get the numerical solutions, and the comparison with analytical solution is fulfilled. The comparison results show that the analytical solutions are satisfactorily precise. At last, the comparisons with other traditional DVAs show that the DVA in this paper can largely reduce the resonance amplitude and broaden vibration frequency range; even it can make the amplitude of the primary system remain small enough in the whole frequency range. 


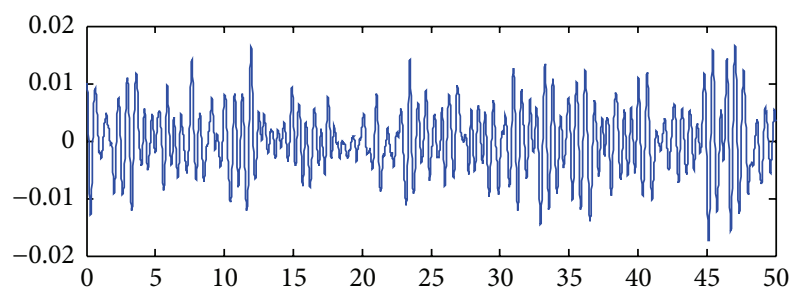

FIGURE 9: The time history of the primary system with DVA by Den Hartog.

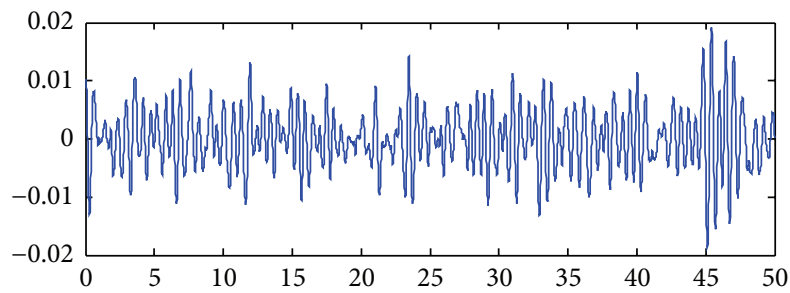

FIGURE 10: The time history of the primary system with DVA by Ren.

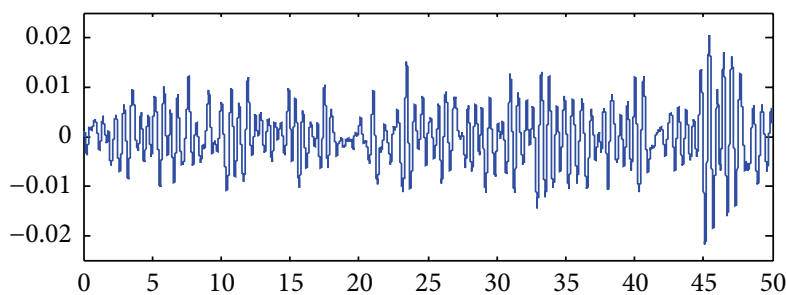

FIgURE 11: The time history of the primary system with DVA by Asami.

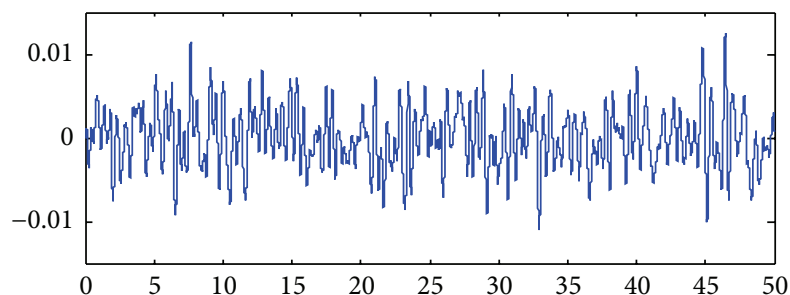

FIGURE 12: The time history of the primary system with DVA in this paper.

The results are useful to design more effective dynamic vibration absorber.

\section{Competing Interests}

The authors declare that there is no conflict of interests regarding the publication of this paper.

\section{Acknowledgments}

The authors are grateful to the support by National Natural Science Foundation of China (no. 11372198), the Cultivation Plan for Innovation team and leading talent in colleges and universities of Hebei province (LJRC018), the Program for Advanced Talent in the universities of Hebei province (GCC2014053), and the Program for Advanced Talent in Hebei province (A201401001).

\section{References}

[1] H. Frahm, "Device for damping vibrations of bodies," U.S. Patent 989,958. 1911.

[2] J. Ormondroyd and J. P. Den Hartog, "The theory of the dynamic vibration absorber," ASME Journal of Applied Mechanics, vol. 50, pp. 9-22, 1928.

[3] E. Hahnkamm, "The damping of the foundation vibrations at varying excitation frequency," Master of Architecture, vol. 4, pp. 192-201, 1932.

[4] J. E. Brock, "A note on the damped vibration absorber," ASME Journal of Applied Mechanics, vol. 13, no. 4, p. A284, 1946.

[5] J. P. Den Hartog, Mechanical Vibrations, McGraw-Hall Book Company, New York, NY, USA, 3th edition, 1947.

[6] D. J. Inman, Vibration with Control, John Wiley \& Sons, London, UK, 2006.

[7] O. Nishihara and T. Asami, "Closed-form solutions to the exact optimizations of dynamic vibration absorbers (minimizations of the maximum amplitude magnification factors)," Journal of Vibration and Acoustics, Transactions of the ASME, vol. 124, no. 4, pp. 576-582, 2002.

[8] T. Asami, O. Nishihara, and A. M. Baz, "Analytical solutions to $H_{\infty}$ and $H_{2}$ optimization of dynamic vibration absorbers attached to damped linear systems," Journal of Vibration and Acoustics, vol. 124, no. 2, pp. 284-295, 2002.

[9] M. Z. Ren, "A variant design of the dynamic vibration absorber," Journal of Sound and Vibration, vol. 245, no. 4, pp. 762-770, 2001.

[10] K. Liu and J. Liu, "The damped dynamic vibration absorbers: revisited and new result," Journal of Sound and Vibration, vol. 284, no. 3-5, pp. 1181-1189, 2005.

[11] T. Asami and O. Nishihara, "Analytical and experimental evaluation of an air damped dynamic vibration absorber: design optimizations of the three-element type model," Journal of Vibration and Acoustics, vol. 121, no. 3, pp. 334-342, 1999.

[12] T. Asami and O. Nishihara, "H2 optimization of the threeelement type dynamic vibration absorbers," Journal of Vibration and Acoustics, Transactions of the ASME, vol. 124, no. 4, pp. 583$592,2002$.

[13] Y.-J. Shen, L. Wang, S.-P. Yang, and G.-S. Gao, "Nonlinear dynamical analysis and parameters optimization of four semiactive on-off dynamic vibration absorbers," Journal of Vibration and Control, vol. 19, no. 1, pp. 143-160, 2013.

[14] Y. J. Shen and M. Ahmadian, "Nonlinear dynamical analysis on four semi-active dynamic vibration absorbers with time delay," Shock and Vibration, vol. 20, no. 4, pp. 649-663, 2013.

[15] R. S. Lakes, "Extreme damping in composite materials with a negative stiffness phase," Physical Review Letters, vol. 86, no. 13, pp. 2897-2900, 2001.

[16] R. S. Lakes, "Extreme damping in compliant composites with a negative-stiffness phase," Philosophical Magazine Letters, vol. 81, no. 2, pp. 95-100, 2002.

[17] R. S. Lakes, T. Lee, A. Bersie, and Y. C. Wang, "Extreme damping in composite materials with negative-stiffness inclusions," Nature, vol. 410, no. 6828, pp. 565-567, 2001. 
[18] R. S. Lakes and W. J. Drugan, "Dramatically stiffer elastic composite materials due to a negative stiffness phase?" Journal of the Mechanics and Physics of Solids, vol. 50, no. 5, pp. 9791009, 2002.

[19] Y. C. Wang and R. S. Lakes, "Extreme stiffness systems due to negative stiffness elements," American Journal of Physics, vol. 72, no. 1, pp. 40-50, 2004.

[20] Y.-C. Wang and R. Lakes, "Negative stiffness-induced extreme viscoelastic mechanical properties: stability and dynamics," Philosophical Magazine, vol. 84, no. 35, pp. 3785-3801, 2004.

[21] Y.-C. Wang and R. Lakes, "Stability of negative stiffness viscoelastic systems," Quarterly of Applied Mathematics, vol. 63, no. 1, pp. 34-55, 2005.

[22] D. L. Platus, "Negative-stiffness-mechanism vibration isolation systems," in Vibration Control in Microelectronics, Optics, and Metrology, 44, vol. 1619 of Proceedings of SPIE, International Society for Optics and Photonics, February 1992.

[23] M. S. Trimboli, R. Wimmel, and E. J. Breitbach, "Quasi-active approach to vibration isolation using magnetic springs," in Smart Structures and Materials 1994: Passive Damping, 73, vol. 2193 of Proceedings of SPIE, May 1994.

[24] S.-T. Park and T.-T. Luu, "Techniques for optimizing parameters of negative stiffness," Proceedings of the Institution of Mechanical Engineers, Part C, vol. 221, no. 5, pp. 505-511, 2007.

[25] T. Mizuno, "Proposal of a vibration isolation system using zeropower magnetic suspension," in Proceedings of the Asia-Pacific Vibration Conference, vol. 2, pp. 423-427, Hangzhou, China, 2001.

[26] T. Mizuno, "Vibration isolation system using zero-power magnetic suspension," in Proceedings of the 15th Triennial World Congress, pp. 955-960, Barcelona, Spain, 2002.

[27] T. Mizuno and Y. Takemori, "A transfer-function approach to the analysis and design of zero-power controllers for magnetic suspension systems," Electrical Engineering in Japan, vol. 141, no. 2, pp. 67-75, 2002.

[28] T. Mizuno, M. Takasaki, D. Kishita, and K. Hirakawa, "Vibration isolation system combining zero-power magnetic suspension with springs," Control Engineering Practice, vol. 15, no. 2, pp. 187196, 2007.

[29] T. Mizuno, T. Toumiya, and M. Takasaki, "Vibration isolation system using negative stiffness," JSME International Journal, Series C: Mechanical Systems, Machine Elements and Manufacturing, vol. 46, no. 3, pp. 807-812, 2003.

[30] M. A. Acar and C. Yilmaz, "Design of an adaptive-passive dynamic vibration absorber composed of a string-mass system equipped with negative stiffness tension adjusting mechanism," Journal of Sound and Vibration, vol. 332, no. 2, pp. 231-245, 2013.

[31] J. Yang, Y. P. Xiong, and J. T. Xing, "Dynamics and power flow behaviour of a nonlinear vibration isolation system with a negative stiffness mechanism," Journal of Sound and Vibration, vol. 332, no. 1, pp. 167-183, 2013.

[32] Y. Zhu, M. Zhang, Z. Liu et al., "A kind of negative stiffness system used in gravity compensation for tiny vibration machines," China Patent 201210537786.1, 2013.

[33] H. Li, P. Zhou, and M. Liu, "A kind of reducing vibration with inhaul cable for passive spring damping negative stiffness damper: China," Patent 201510059994.9, 2015.

[34] H. B. Peng, Y. J. Shen, and S. P. Yang, "Parameters optimization of a new type of dynamic vibration absorber with negative stiffness," Chinese Journal of Theoretical and Applied Mechanics, vol. 47, no. 2, pp. 320-327, 2015. 


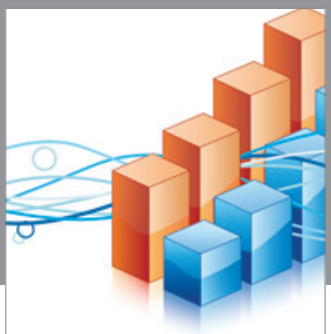

Advances in

Operations Research

vatem alat4

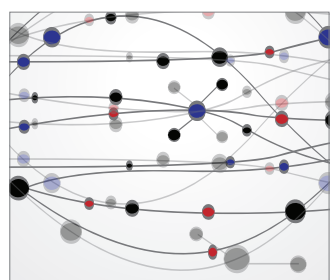

\section{The Scientific} World Journal
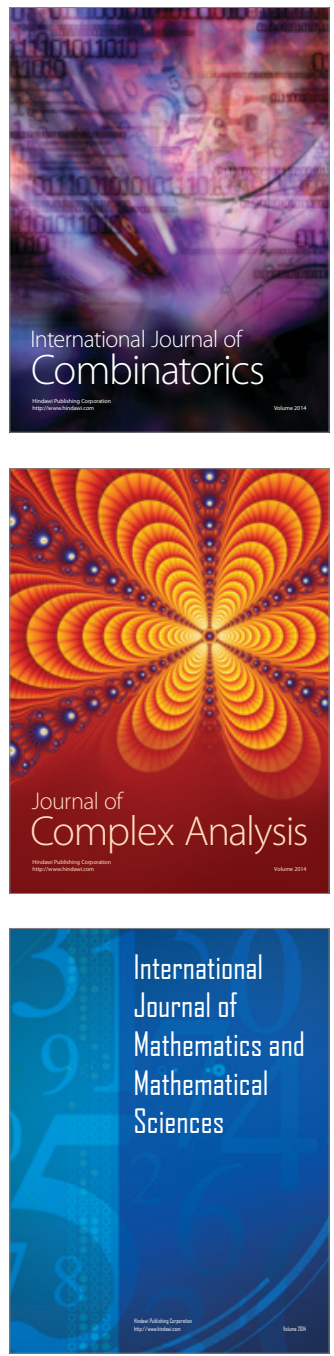
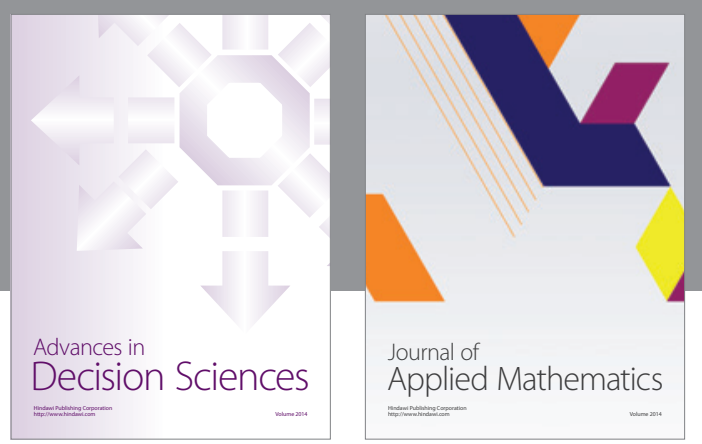

Algebra

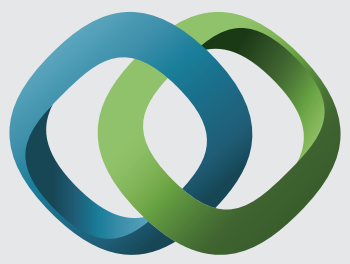

\section{Hindawi}

Submit your manuscripts at

http://www.hindawi.com
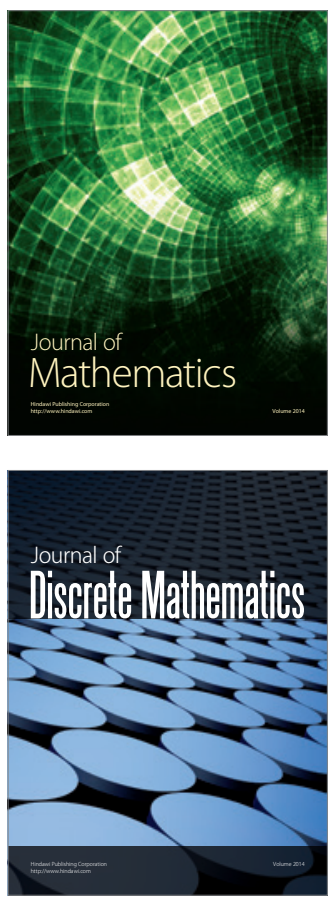

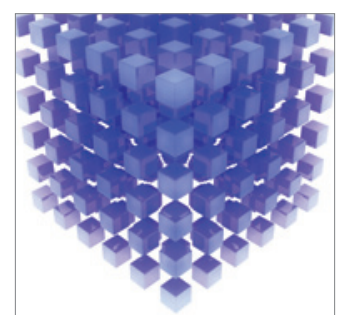

Mathematical Problems in Engineering
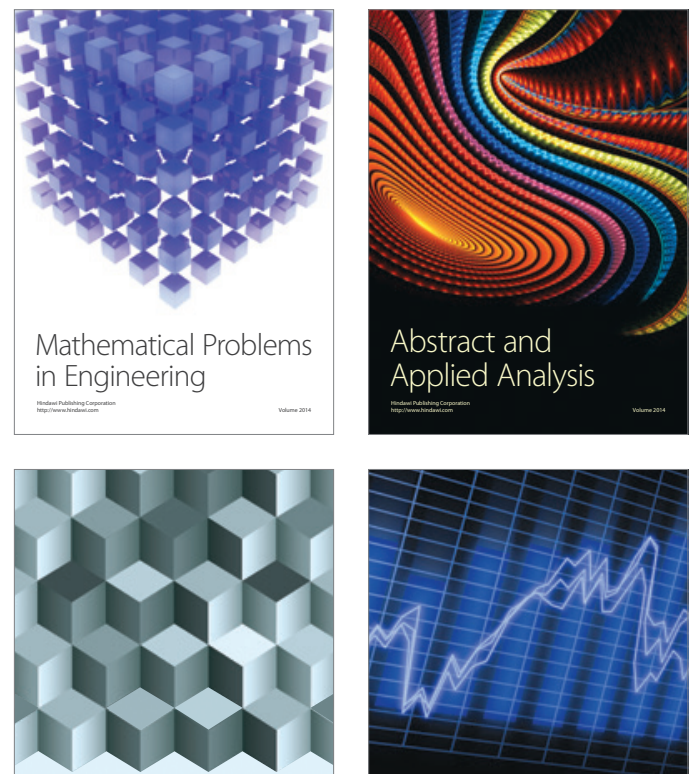

Journal of

Function Spaces

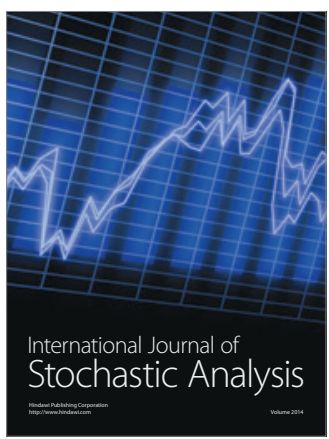

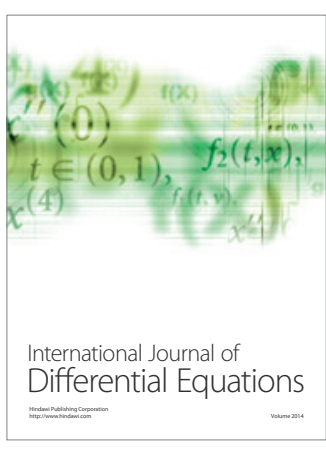
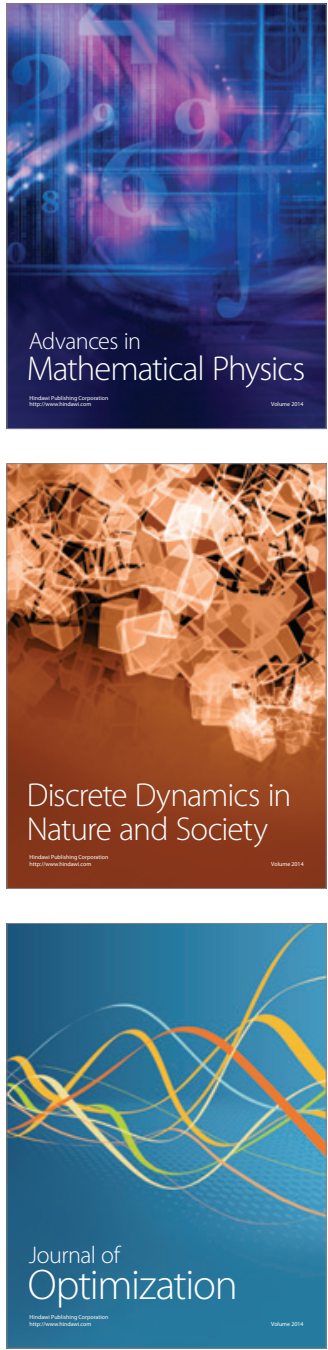